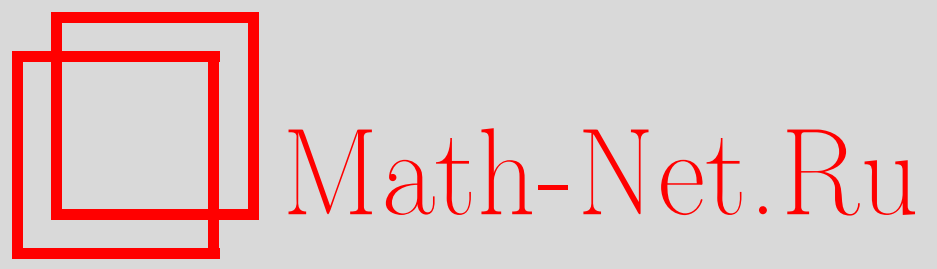

В. П. Орлов, О модели Олдройда вязкоупругой жидкости, Функи. анализ и его прил., 1999, том 33, выпуск 1, 83-87

DOI: https://doi.org/10.4213/faa345

Использование Общероссийского математического портала MathNet.Ru подразумевает, что вы прочитали и согласны с пользовательским соглашением

http://www.mathnet.ru/rus/agreement

Параметры загрузки:

IP : 44.207 .124 .84

26 апреля 2023 г., 16:16:18

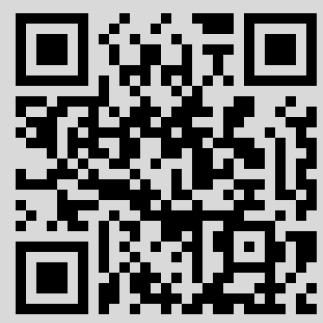


Отметим, что трудности с доказательством существования, единственности и непрерывной зависимости от параметра точных периодических решений задачи (1) связаны с невыполнением условия невырожденности Пуанкаре, состоящего в отсутствии в окрестности точки $1 \in \mathbb{C}$ мультипликаторов, т.е. точек спектра оператора монодромии, отвечающего периодическому решению $\zeta_{E \varepsilon}(t)$ упрощенного уравнения. (Монодромия рассматривается на поверхности уровня функции Гамильтона $\bar{H}$.) По-видимому, это не просто технические трудности, и все эти патологии, т.е. несуществование, неединственность и отсутствие непрерывной зависимости, при невыполнении условия Пуанкаре могут реализовываться. В нашем случае причиной вырождения по Пуанкаре является неограниченность частот линейной задачи $(1)_{\varepsilon=0}$, в результате чего при всех значениях параметров единица оказывается предельной точкой для множества мультипликаторов, отвечающих решениям $\zeta_{E \varepsilon}(t)$. Таким образом, эти патологии — целиком эффект бесконечномерности рассматриваемой динамической системы, определяемой уравнением (1), точнее, они не типичны в конечномерном случае, и их можно назвать «размыванием» точных периодических решений линейных мод при нелинейном возмущении.

Заметка написана на основе докладов, прочитанных автором весной 1996 года на проходивших в МГУ заседаниях «Чебышёвских чтений» и конференции имени И. Г. Петровского.

\title{
ЛИТЕРАТУРА
}

1. Bambusi D., Giorgilli A. J. Statist. Phys., 71, 569-606 (1993). 2. Bambusi D., Nekhoroshev N. N. Phys. D, 122, 73-104 (1998). 3. Craig W., Wayne C. E. Comm. Pure Appl. Math., 46, 1409-1501 (1993). 4. Kuksin S. B. Lect. Notes Math., vol. 1556, Springer-Verlag, 1994. 5. Rabinowitz P. H. Comm. Pure Appl. Math., 31, 31-68 (1978).

Московский государственный университет, механико-математический факультет

Поступило в редакцию 29 декабря 1997 г.

УДК 517.98.63, 517.984.5

\section{О модели Олдройда вязкоупругой жидкости *}

\author{
(c) 1999. В. П. Орлов
}

1. Рассматривается модель вязкоупругой жидкости Олдройда [1]

$$
\begin{aligned}
& v_{t}(t, x)+D(v)-\gamma_{1} \Delta v(t, x) \\
& -\gamma_{2} \Delta \int_{0}^{t} \exp (\alpha(s-t)) v(s, x) d s+\nabla p(t, x)=f(t, x), \\
& \operatorname{div} v(t, x)=0,(t, x) \in Q, \quad \int_{\Omega} p(t, x) d x=0, t \geqslant 0 \\
& v(0, x)=v_{0}(x), x \in \Omega, \quad v(t, x)=0, t \geqslant 0, x \in \partial \Omega .
\end{aligned}
$$

* Работа выполнена при частичной поддержке РФФИ, грант 96-01-01639, и ISF, грант RJ3300. 
Здесь $\gamma_{1}>0, \gamma_{2}, \alpha \in \mathbb{R}, D(v)=\sum_{k=1}^{n} v_{k} \partial v / \partial x_{k}, Q=[0, \infty) \times \Omega, \Omega$ ограниченная область в $\mathbb{R}^{n}, n \geqslant 2$, с границей $\partial \Omega$ класса $C^{2}$. В [1] установлена разрешимость задачи $(1)$ в $L_{2}(\Omega)$ при необходимых условиях на данные, локальная (по $t$ ) при $n=3$ и нелокальная при $n=2$. Поведение решений на бесконечности при различных ограничениях на данные, бо́льших, чем необходимые, изучалось в [2-7], причем в [7] установлена устойчивость стационарных решений относительно возмущений правых частей из некоторых гёльдеровых (по времени) пространств. Ниже мы устанавливаем разрешимость задачи (1) в классах Соболева при необходимых условиях для малых данных. Полученные оценки решений означают устойчивость нулевого решения в сильных нормах.

Введем необходимые обозначения. Обозначим через $S_{q}$ замыкание в метрике пространства $L_{q}(\Omega)$ множества определенных на $\Omega$ финитных соленоидальных вектор-функций. Тогда оператор $\mathscr{P}$ ортогонального проектирования в $L_{2}(\Omega)$ на $S_{2}$ является ограниченным и в $L_{q}(\Omega)$ при $1<q<\infty$ (см. [8]). Рассмотрим в $S_{q}$ оператор $\widetilde{\Delta}=-\mathscr{P} \Delta$, заданный на области определения $D(\widetilde{\Delta})=W_{q}^{2}(\Omega) \cap \stackrel{\circ}{W} \underset{q}{1}(\Omega) \cap S_{q}$. Здесь и далее через $W_{q}^{k}(\Omega)$ обозначаются пространства Соболева функций, определенных на $\Omega$, а через $W_{q}^{k, m}(Q)$ пространства Соболева определенных на $Q$ функций, имеющих производные по $t$ до порядка $k$ и производные по $x$ до порядка $m$, суммируемые со степенью $q$. Нормы в $W_{q}^{k}(\Omega), W_{q}^{k, m}(Q), L_{q}(\Omega)$ и в $L_{q}(Q)$ обозначаются через $|\cdot|_{k}$, $\|\cdot\|_{k, m},|\cdot|_{0}$ и $\|\cdot\|_{0}$ соответственно. Обозначим через $L_{q, \varkappa}(Q)$ пространство функций, для которых конечна норма $\|\phi\|_{0 ; \varkappa}=\|\exp (\varkappa t) \phi(t, x)\|_{0}$, а через $W_{q, \varkappa}^{k, m}(Q)$ пространство функций, для которых конечна норма $\|\phi\|_{k, m ; \varkappa}=$ $\|\exp (\varkappa t) \phi(t, x)\|_{k, m}$, и пусть $W=S_{q} \cap W_{q}^{2-2 / q}(\Omega) \cap \stackrel{\circ}{W}{ }_{q}^{1}(\Omega)$. Пусть $0<\sigma_{1}<$ $\sigma_{2}<\cdots<\sigma_{n}<\ldots-$ спектр оператора $\widetilde{\Delta}$ (см. [8]), а функции $\lambda_{k}(\sigma)$ и $\beta_{k}(\sigma), k=1,2$, переменной $\sigma$ определяются формулами

$$
\lambda_{k}(\sigma)=-\frac{1}{2}\left(\left(\gamma_{1} \sigma-\alpha\right)-(-1)^{k}\left(\left(\gamma_{1} \sigma-\alpha\right)^{2}-4 \gamma_{2} \sigma\right)^{1 / 2}\right), \quad \beta_{k}(\sigma)=\lambda_{k}(\sigma)-\alpha .
$$

Сформулируем основной результат.

Теорема 1. Пусть $q \in((n+2) / 3,+\infty)$. Пусть выполняютсл условия

$$
\gamma_{1} \sigma_{1}+\alpha>0, \quad \gamma_{1} \alpha+\gamma_{2}>0, \quad \varkappa<\varkappa_{0}=\min \left(\gamma_{2} / \gamma_{1}+\alpha,-\operatorname{Re} \beta_{2}\left(\sigma_{1}\right)\right)
$$

$u \varkappa \geqslant 0$. Тогда найдется такое $\delta>0$, что при всех $f \in L_{q, \varkappa}(Q), v_{0} \in W$, таких, что $\|f\|_{0 ; \varkappa} \leqslant \delta,\left|v_{0}\right|_{2-2 / q} \leqslant \delta$, задача (1) имеет единственное решение $(v(t, x), p(t, x))$, где $v(t, x) \in W_{q, \varkappa}^{1,2}(Q), p(t, x) \in W_{q, \varkappa}^{0,1}(Q)$, причем

$$
\begin{aligned}
\|v\|+\|p\|_{0,1 ; \varkappa} & \equiv\left(\|v\|_{1,2 ; \varkappa}+\left\|\int_{0}^{t} \exp (\alpha(s-t)) v(s, x) d s\right\|_{0,2 ; \varkappa}\right. \\
& \left.\quad+\sup _{t \geqslant 0}|\exp (\varkappa t) v(t, x)|_{2-2 / q}\right)+\|p\|_{0,1 ; \varkappa} \\
& \leqslant M_{1}\left(\|f\|_{0 ; \varkappa}+\left|v_{0}\right|_{2-2 / q}\right) .
\end{aligned}
$$

Доказательство теоремы 1 проводится сведением задачи (1) к некоторому 
нелинейному операторному уравнению путем обращения его линейной части, которая рассматривается как абстрактная задача Коши.

2. Рассмотрим сначала в произвольном банаховом пространстве $E$ линейную задачу Коши

$$
v^{\prime}(t)+\gamma_{1} A v(t)+\gamma_{2} A \int_{0}^{t} \exp (\alpha(s-t)) v(s) d s=f(t), \quad t \geqslant 0, \quad v(0)=v_{0} .
$$

Пусть $A$ сильно позитивен в $E$ (см. [9]), а его спектр $\Sigma$ равен $\Sigma_{1} \cup \Sigma_{2}$, причем $\Sigma_{1}$ состоит из собственных значений $0<\sigma_{1}<\sigma_{2}<\cdots<\sigma_{N}$, которым соответствуют конечномерные подпространства, а $\Sigma_{2}$ лежит в пересечении полуплоскости $\operatorname{Re} \lambda>\tilde{\sigma}_{N}$ и сектора $|\operatorname{Arg} \lambda| \leqslant \psi_{0}$, где $\tilde{\sigma}_{N}>$ $\sigma_{N}$, а $\psi_{0} \in(0, \pi / 2)$. Изучим разрешимость задачи (4) в банаховом пространстве $L_{q, \varkappa}(0,+\infty ; E)$ функций, для которых конечна норма $\|\phi\|_{L_{q, \varkappa}}=$ $\|\phi(t) \exp (\varkappa t)\|_{L_{q}(0, \infty ; E)}$. Нам понадобится

УСловие 1 . Интегральный оператор $R_{\mu}(f)=A \int_{0}^{t} T_{0}(\mu(t-s)) f(s) d s$ ограничен в $L_{q_{0}}(0,+\infty ; E)$ при некотором $1<q_{0}<+\infty$, и $\mu=1$.

Здесь $T_{0}(t)=\exp (-t A)$ - аналитическая полугруппа, порожденная оператором $A$ (см. [9]). Условие 1 эквивалентно коэрцитивной разрешимости задачи

$$
v^{\prime}(t)+A v(t)=f(t), \quad v(0)=0
$$

(см. [10]).

Определим $E_{1-1 / q}$ как совокупность элементов из $E$, для которых конечна норма $] v\left[1-1 / q=\left(\int_{0}^{+\infty}\left\|T_{0}(t) v\right\|^{q} d t\right)^{1 / q}\right.$.

ТеОРема 2. Пусть $\tilde{\sigma}_{N}$ достаточно велико. Пусть выполняются условие 1 и неравенства (2). Тогда для любых $f \in L_{q, \varkappa}(0,+\infty ; E)$ u $v_{0} \in E_{1-1 / q}$ задача (4) однозначно разрешима и справедливо неравенство

$$
\begin{aligned}
\left\|v^{\prime}(t)\right\|_{L_{q, \varkappa}} & +\left\|\int_{0}^{t} \exp (\alpha(s-t)) v(s, x) d s\right\|_{L_{q, \varkappa}} \\
& \left.+\sup _{t \geqslant 0}\right] \exp (\varkappa t) v(t)\left[_{1-1 / q}+\|A v\|_{L_{q, \varkappa}} \leqslant M_{2}\left(\|f\|_{L_{q, \varkappa}}+\right] v_{0}[1-1 / q) .\right.
\end{aligned}
$$

Для доказательства задача (4) разбивается на задачи $\mathscr{L}_{i}, i=1,2$, $v_{i}^{\prime}(t)+\gamma_{1} A_{i} v_{i}(t)+\gamma_{2} A_{i} \int_{0}^{t} \exp (\alpha(s-t)) v_{i}(s) d s=f_{i}(t), \quad t \geqslant 0, \quad v_{i}(0)=v_{0 i}$ в пространствах $L_{q, \varkappa}\left(0,+\infty ; E_{i}\right)$. Здесь $A_{i}=P_{i} A, v_{i}=P_{i} v, E_{i}=P_{i} E$, $v_{0 i}=P_{i} v_{0}, f_{i}=P_{i} f$, a $P_{i}$ являются проекторами Рисса на подпространства $E_{i}$ в $E$, порожденные разбиением спектра $\Sigma$ на $\Sigma_{1}$ и $\Sigma_{2}$. Отметим, что задача $\mathscr{L}_{1}$ является задачей Коши для распадающейся системы $N$ обыкновенных интегро-дифференциальных уравнений, а задача $\mathscr{L}_{2}$ остается задачей с неограниченными операторными коэффициентами.

Вначале результат теоремы 2 устанавливается для задачи $\mathscr{L}_{2}$. Для этого ее решение выписывается в виде 


$$
\begin{array}{r}
v_{2}(t)=\mathscr{A}_{1}\left(\mathscr{A}_{1}-\mathscr{A}_{2}\right)^{-1} \int_{0}^{t} T_{1}(t-s) \\
f_{2}(s) d s+\mathscr{A}_{2}\left(\mathscr{A}_{2}-\mathscr{A}_{1}\right)^{-1} \int_{0}^{t} T_{2}(t-s) f_{2}(s) d s \\
+\left(\mathscr{A}_{1} T_{1}(t)-\mathscr{A}_{2} T_{2}(t)\right)\left(\mathscr{A}_{1}-\mathscr{A}_{2}\right)^{-1} v_{02} . \quad(6)
\end{array}
$$

Здесь $\mathscr{A}_{i}, i=1,2$, суть корни характеристического уравнения

$$
\lambda^{2}+\left(\gamma_{1} A_{2}-\alpha I\right) \lambda+\gamma_{2} A_{2}=0
$$

а $T_{i}(t)=\exp \left(t\left(\mathscr{A}_{i}-\alpha I\right)\right)$. Используя результаты работ [11-13], можно показать, что при достаточно большом $\tilde{\sigma}_{N}$ оператор $\mathscr{A}_{2}$ неограничен, оператор $\mathscr{A}_{1}$ ограничен, а аналитическая полугруппа $T_{2}(t)$ и группа $T_{1}(t)$ таковы, что $\left\|T_{i}(t)\right\| \leqslant M \exp \left(-t \rho\left(\tilde{\sigma}_{N}\right)\right)$, где $t \geqslant 0$ и $\rho(t) \rightarrow+\infty$ при $t \rightarrow+\infty$. Так как

$$
\mathscr{A}_{2}=-\frac{1}{2}\left(I+\left(I-4 \gamma_{2} A_{2}\left(\gamma_{1} A_{2}-\alpha I\right)^{-2}\right)^{1 / 2}\right)\left(\gamma_{1} A_{2}-\alpha I\right) \equiv-\frac{1}{2} B\left(\gamma_{1} A_{2}-\alpha I\right),
$$

то с помощью теоремы об отображении спектра и выбора $\tilde{\sigma}_{N}$ достаточно большим получаем, что спектр ограниченного оператора $B$ лежит строго в правой полуплоскости. В силу формул Коши-Рисса

$$
T_{2}(t)=\frac{1}{2 \pi i} \int_{\Gamma} P_{2} T_{0}\left(\frac{1}{2} \gamma_{1} \mu t\right) \exp \left(\left(\frac{1}{2} \mu-1\right) \alpha t\right)(\mu I-B)^{-1} d \mu .
$$

Здесь контур $\Gamma$ лежит внутри сектора аналитичности полугруппы $T_{0}(t)$ и охватывает спектр оператора $B$. Отсюда с помощью обобщенного неравенства Минковского получаем, что доказательство оценки (5) сводится к вытекающей из условия 1 равномерной по $\mu$ оценке интегрального оператора $R_{\mu}$ в весовом пространстве $L_{q, \varkappa}\left(0, \infty ; E_{2}\right)$ (см. [13]).

Решение задачи $\mathscr{L}_{1}$ выписывается в виде $N$-мерного вектора $v_{1}(t)$ с компонентами $v_{1 k}(t)$ вида (6) при $\mathscr{A}_{k}=\beta_{k}\left(\sigma_{k}\right)$ в случае, если корни $\lambda_{k}$ характеристического уравнения $(\chi)$ для $A_{2}=\sigma_{k}$ простые, и с естественными изменениями в $(6)$ в случае кратных корней. Число $\varkappa_{0}$ при этом дает оценку показателя экспоненциального убывания на $+\infty$ для $T_{1}(t)$. Оценка $(5)$ для $\mathscr{L}_{2}$ очевидна.

3. Рассмотрим теперь линейную задачу

$$
\begin{gathered}
v_{t}(t, x)+\gamma_{1} \widetilde{\Delta} v(t, x)+\gamma_{2} \widetilde{\Delta} \int_{0}^{t} \exp (\alpha(s-t)) v(s, x) d s=\varphi(t, x), \\
(t, x) \in Q, \quad v(0, x)=v_{0}(x), x \in \Omega, \quad v(t, x)=0, t \geqslant 0, x \in \partial \Omega .
\end{gathered}
$$

ТЕОРема 3. Если неравенства (2) выполняются для любых $\varphi \in L_{q, \varkappa}(Q)$ $u v_{0} \in W$, то задача (7) имеет единственное решение и справедлива оченка

$$
\|v\| \leqslant M_{3}\left(\|\varphi\|_{0 ; \varkappa}+\left|v_{0}\right|_{2-2 / q}\right) .
$$

Для доказательства теоремы 3 задача (7) сводится к задаче (4) при $E=S_{q}$ и $A=\widetilde{\Delta}$ с использованием результатов из $[8,12]$.

4. Перейдем к доказательству теоремы 1. Записывая решение задачи (7) как $v=\mathscr{N}\left(v_{0}, \varphi\right)$ и полагая $\varphi=\mathscr{P} f-\mathscr{P} D(v)$, сведем задачу $(1)$ к операторному уравнению

$$
v=\Phi(v) \equiv \mathscr{N}\left(v_{0}, \mathscr{P} f\right)+\mathscr{N}(0, \mathscr{P} D(v))
$$


Используя оценку (8), квадратичный характер нелинейности $D(v)$ и подходящие теоремы вложения для анизотропных соболевских пространств, получаем, что при достаточно «малых» $v_{0}$ и $f$ оператор $\Phi$ переводит в себя шар $B\left(R_{0}\right)=\left\{v:\|v\|_{1,2} ; \varkappa \leqslant R_{0}\right\}$ при некотором малом $R_{0}$ и является сжимающим. Оценка (3) для части $v$ решения задачи (1) вытекает из оценки (8) и уравнения $(*)$. Так же, как и в [8], по соленоидальной функции $v$ находится функция давления $p$, а ее оценка следует из оценки $v$ и уравнения (1).

ЗАмЕчАНИЕ. Можно показать, что невыполнение условий (2) вызывает некоторого типа (см. [8]) неустойчивость нулевого решения задачи (1), так как тогда либо оператор $\mathscr{A}_{1}$ оказывается неограниченным, либо в спектре оператора $\mathscr{A}_{2}-\alpha I$ появляются точки с положительной вещественной частью. Заметим также, что, в отличие от [7], коэффициенты $\gamma_{2}$ и $\alpha$ могут быть отрицательными, а $q<2$. При этом $\operatorname{Re} \beta_{2}\left(\sigma_{1}\right)$ может быть отрицательным числом, лишь если $\alpha<0$, а $\sigma_{1}<-\alpha / \gamma_{1}$. Из определения решения (1), оценки (3) и $L_{q}$-теории для задачи $(\mathrm{Z})$ при $A=\widetilde{\Delta}$ (см. [8]) следует необходимость выбора классов правых частей и начальных данных для (1) в соответствии с теоремой 1 .

\section{ЛитеРАТУРА}

1. Агранович Ю. Я., Соболевский П. Е. ДАН УССР, сер. А., № 10, 3-6 (1989). 2. Котсиолис А. А., Осколков А. П. Записки научн. сем. ЛОМИ, 150, № 6, 48-52 (1986). 3. Каразеева Н. А. Записки научн. сем. ЛОМИ, 156, № 16, 69-72 (1986). 4. Осколков А. П. Труды МИАН, 179, № 13, 126-164 (1988). 5. Осколков А. П. Функциональные методы в теории нестационарных течений линейных вязкоупругих жидкостей. Препринт ЛОМИ р-2-83. 6. Каразеева Н. А., Котсиолис А. А., Осколков А. П. Труды МИАН, 188, № 14, 59-87 (1990). 7. Sobolevskii P. E. Differential Integral Equations, 7, No. 6, 1597-1612 (1994). 8. Юдович В. И. Метод линеаризации в гидродинамической теории устойчивости. Изд-во Ростовского унта, Ростов, 1984. 9. Красносельский М. А., Забрейко П. П., Пустьльник Е. И., Соболевский П. Е. Интегральные операторы в пространствах суммируемых функций. Наука, М., 1966. 10. Соболевский П. Е. ДАН СССР, 157, № 1, 51-55 (1964). 11. Orlov V. P. Differential Integral Equations, 4, No. 1, 89-101 (1991). 12. Orlov V. P. Nonlinear Anal., 26, No. 12, $1937-1950$ (1996). 13. Орлов В. П. Дифференц. уравн., № $2,272-280$ (1976).

Воронежский государственный университет e-mail: orlov@kfa.vsu.ru

Поступило в редакцию 22 марта 1997 г.

\section{Об уравнениях с непрерывными отображениями в банаховых пространствах}

(C) 1999. К. Н. Солтанов

Работа посвящена изучению некоторых свойств непрерывных отображений, действующих в банаховых пространствах, и уравнений (включений) с такими отображениями. В ней используются соображения, приведенные в [1]. 